\title{
4. Bewerten und Gewichten: Evidenz als Entscheidungshilfe in der Gesundheits- und Umweltpolitik
}

\author{
Christine Haßauer, Sarah Ehlers und Jutta Roosen
}

Sind wir bereit aus Profitgründen [...] Artensterben, Bodendegradierung und nitrithaltiges Trinkwasser zu akzeptieren? ${ }^{1}$

Evidenz als Entscheidungshilfe ist ein zentrales Element für Regulierungen und Interventionen in pluralen Gesellschaften. Öffentliche Entscheidungen sind komplexe Vorgänge, schließlich gilt es nicht nur eine Vielzahl möglicher Folgen, sondern auch unterschiedliche Interessen zu berücksichtigen. Insbesondere staatliche Eingriffe, die die Freiheit von Einzelnen einschränken, sind in demokratischen Gesellschaften zu begründen. Dafür haben sich Verfahren - von uns im Folgenden als Evidenzpraktiken verstanden - etabliert, mit denen die Auswirkungen von Entscheidungen in systematisierter Form mithilfe von Messgrößen und formalisierter Kriterien antizipiert werden. ${ }^{2}$ Insbesondere die Kosten-Nutzen-Analyse konnte sich als zentrale Praxis ökonomisierter Entscheidungsprozesse durchsetzen, anhand derer die Folgen staatlicher Eingriffe bewertet und gewichtet werden. Dieses Kapitel wird diese Bewertungs- und Gewichtungsprozesse als Element der evidenzbasierten Politik betrachten. Dazu werden wir immer wieder auf zwei Fallstudien rekurrieren, die Regulierung von Pestiziden sowie international gesteuerte Ansätze zur globalen Bekämpfung von Malaria, um die Praktiken zu veranschaulichen.

Gerade in einer Zeit des wachsenden Einflusses sogenannter „postfaktischer" Geltungsbehauptungen, des Erstarkens der Populisten und der zugehörigen Kommunikationsstrategien in den Medien verspricht der Bezug auf Evidenz, also die Mobilisierung von gesellschaftlich anerkannten Da-

1 Volksbegehren Artenvielfalt: https://volksbegehren-artenvielfalt.de/ [Stand: 22.6.2019].

2 In der Europäischen Union sind zum Beispiel Standardindikatoren für evidenzbasierte Entscheidungen in den Bereichen nachhaltiges Wachstum, Sozialpolitik oder Währung definiert. Auch die Millenium Development Goals der Vereinten Nationen basieren auf einer Sammlung statistischer Indikatoren, anhand derer die evidenzbasierte Umsetzung von armutsbekämpfenden und auf die Nachhaltigkeit bezogenen Maßnahmen analysiert werden können. Statistisches Bundesamt, „Indikatoren für evidenzbasierte Politik“ 2019, Wiesbaden. 
ten und Fakten, auf eine gemeinsame Entscheidungsbasis zurückzukehren. Durch Evidenz lassen sich folglich Entscheidungen zu kontroversen Themen rechtfertigen und Entscheidungsprozesse rationalisieren. ${ }^{3}$ Von evidenzbasierter Politik wird erwartet, dass sie Ideologien und Machtasymmetrien in der Entscheidungsfindung neutralisiert. ${ }^{4}$ Gleichzeitig verspricht die wissensbasierte Legitimation politischer Maßnahmen eine Erhöhung der Wirksamkeit dieser Maßnahmen sowie eine bessere Ressourcennutzung und eine geringere Fehleranfälligkeit. ${ }^{5}$

Ein zentrales und kritisches Element der evidenzbasierten Entscheidung ist die Abwägung zwischen verschiedenen Auswirkungen und Interessen. So betreffen politische Entscheidungen nicht nur einen Aspekt in der Lebensumwelt der Menschen, sondern ganz unterschiedliche Bereiche. Die Zulassung eines Pestizids wirkt sich nicht nur auf die Gesundheit von Anwendern und Konsumenten aus, sondern auch auf die Biodiversität, Wirtschaft und Verteilung von Vermögen und Umweltresourcen. Dabei sind diese Auswirkungen für verschiedene Akteure ungleich relevant, wie sich auch in Debatten um Pestizidzulassungen zeigt: Wenn beispielsweise der Einsatz eines Pestizids mit dem Verschwinden bestimmter Arten, aber auch mit der Steigerung der landwirtschaftlichen Produktivität einhergeht, ist davon auszugehen, dass seine Zulassung von Naturschutzgruppen und Landwirten unterschiedlich beurteilt wird. Um evidenzbasiert demokratische Entscheidungen treffen zu können, sind solche Auswirkungen daher nicht nur zu messen oder zu ermessen, ${ }^{6}$ sondern sie sind auch zu bewerten und zu gewichten, insbesondere da an diese Entscheidungen oft auch die Verteilung von Ressourcen geknüpft ist. Damit werden Bewertung

3 Andrea Saltelli u. Mario Giampietro, What Is Wrong with Evidence Based Policy, and How Can It Be Improved?, in: Futures 91, 2017, S. 62-71; Mark J. Dobrow, Vivek Goel u. Ross E.G. Upshur, Evidence-Based Health Policy. Context and Utilisation, in: Social Science and Medicine 58, H. 1, 2004, S. 207-217.

4 Kari Lancaster, Kathryn Leigh Seear, Carla Treloar u. Alison Ritter, The Productive Techniques and Constitutive Effects of "Evidence-Based Policy" and "Consumer Participation" Discourses in Health Policy Processes, in: Social Science and Medicine 176, 2017, S. 60-68.

5 Evidence-Based Policymaking Collaborative, Principles of Evidence-Based Policymaking, 2019: https:/www.evidencecollaborative.org/; European Commission, EACEA u. Eurydice, Support Mechanisms for Evidence-Based Policy-Making in Education, Luxemburg 2017; Michael G. Marmot, Evidence Based Policy or Policy Based Evidence?, in: British Medical Journal 328, April 2004, S. 906-907.

6 Eine ausführlichere Abhandlung dieser Praktiken ist im Kapitel Messen und Ermessen dieses Buches zu finden. 
und Gewichtung zur Basis für den Austausch von Argumenten und zu wichtigen Bestandteilen im Modus der Konfliktbewältigung.

Im Folgenden werden die Evidenzpraktiken Bewerten und Gewichten, die der Findung und Begründung von Entscheidungen dienen, kritisch diskutiert. Dabei wendet sich das Kapitel im zweiten Abschnitt der Beschreibung der ökonomischen Praktik der Monetarisierung von Effekten (Bewertung) sowie deren Abwägung (Gewichtung) im Rahmen der KostenNutzen-Analyse zu. Dabei wird ausgeführt, wie in der Kosten-Nutzen-Analyse zum Beispiel gesundheitliche Auswirkungen in monetäre Werte überführt und gegen wirtschaftliche Ertragsverluste gewichtet werden können, wodurch eine neue Form von (ökonomischer) Evidenz entsteht.

Im dritten Abschnitt betrachtet das Kapitel die Etablierung der KostenNutzen-Analyse als formalisierte Form der Bewertung und Gewichtung in Entscheidungsprozessen. Die Kosten-Nutzen-Analyse trug maßgeblich dazu bei, dass sich die Praktiken von Bewertung und Gewichtung langsam vom nicht kodifizierten „Ausdiskutieren“ mit unklaren Verantwortlichkeiten zu einem idealerweise transparenten, nachvollziehbaren Evaluierungsund Entscheidungsprozess entwickelte. Diese Entwicklung geschah vor dem Hintergrund gewachsener gesellschaftlicher Ansprüche an politische Transparenz sowie der Globalisierung, die die Harmonisierung und Vergleichbarkeit politischer Maßnahmen erfordert.

Der vierte Abschnitt zeigt beispielhaft an der Roll-Back-Malaria-Initiative (RBM), wie ökonomisierte Entscheidungsprozesse internationaler Organisationen und insbesondere die Kosten-Nutzen-Analyse in einem Politikfeld wirken, das entscheidend von konkreten, lokalen Bedingungen globaler Armut geprägt ist. Im Mittelpunkt steht dabei die lokale Anwendung internationaler Vorgaben und den Problemen kontextspezifischer Evidenz. Auch wird die Übertragung allgemeiner Parameter und Determinanten auf die verarmten Regionen der Welt diskutiert.

Zuletzt sollen kritische Aspekte der Kosten-Nutzen-Analyse diskutiert werden. Dies ermöglicht eine differenziertere Betrachtung der Evidenzpraktiken Bewerten und Gewichten. Weiterhin werden die Evidenzpraktiken in einen größeren Zusammenhang gestellt und es wird die Frage aufgeworfen, inwieweit durch diese Verfahren Transparenz geschaffen wird und nicht zuletzt, inwieweit damit Evidenz, verstanden als eine Qualität von Wissen, in Entscheidungsprozessen erzeugt und nutzbar gemacht werden kann. 
Bewerten und Gewichten als Evidenzpraktiken zur Begründung von Entscheidungen

Bewertungen und Gewichtungen sind vor allem dann relevant (aber auch umstritten), wenn Entscheidungen komplexe Effekte auf unterschiedliche Akteure implizieren. Am im Folgenden diskutierten Fallbeispiel, der Zulassung von Pestiziden, lassen sich gerade aufgrund der Komplexität von Effekten die Praktiken Bewerten und Gewichten besonders deutlich illustrieren: Entscheidungen in der Pestizidregulierung können eine Vielzahl von ökologischen, ökonomischen und sozialen Konsequenzen aufweisen, die in ihren einzelnen Dimensionen zu bewerten und zu gewichten sind. Ein großes Spektrum von Pestiziden dient in der Produktion von Nahrungsmitteln einerseits der Erhöhung der Produktivität und Qualität, sorgt für die Vermeidung von Resistenzen und vermindert das Produktionsrisiko. Andererseits verschmutzen Pestizide die Umwelt und bergen ein Gesundheitsrisiko für Anwender und Konsumenten. ${ }^{7}$ Beide Evidenzpraktiken, $B e$ werten und Gewichten, dienen der Darstellung und im Zweifel der Rechtfertigung, warum bestimmte Entscheidungen, zum Beispiel die Zulassung von Pestiziden, getroffen werden und beschreiben daher Modi des Begründens mit dem Ziel der Konfliktbewältigung. Die Praktiken sind hierbei nicht als Gegensatzpaar zu sehen, sondern ergänzen sich: In der Zulassung eines Pestizids muss einer Gewichtung der Evidenz zu den Wirkungen in unterschiedlichen Dimensionen, wie zum Beispiel der ökologischen oder gesundheitlichen Folgen, immer eine Bewertung der einzelnen Folgen vorangehen. Eine umfassende Beurteilung eines Pestizids ist meist nur durch eine Gewichtung verschiedener Konsequenzen möglich.

Die Praktik des Bewertens nimmt eine Wertzuschreibung für einen einzelnen Effekt einer Entscheidung vor. Dies geschieht in Kosten-NutzenAnalysen durch Monetarisierung, also die Zuschreibung eines Geldwertes. Dazu stehen unterschiedliche Methoden der ökonomischen Bewertung zur Verfügung. Während für Marktgüter wie Getreide Marktpreise für die Zuschreibung eines Wertes herangezogen werden können, verlangen Umweltressourcen wie Böden oder Gesundheit nach anderen Bewertungsverfahren. Beispielsweise wird in der Bewertung von Leben und Gesundheit der Wert eines statistischen Lebens (Value per Statistical Life, VSL) als Bewertungseinheit herangezogen. Somit geht es also nicht um die Gefähr-

7 Steven E. Sexton, Zhen Lei u. David Zilberman, The Economics of Pesticides and Pest Control, in: International Review of Environmental and Resource Economics 1, H. 3, 2007, S. 271-326. 
dung eines identifizierten individuellen Lebens, sondern um die Veränderung des Krankheitsrisikos oder der Sterberate. Die damit einhergehenden volkswirtschaftlichen Kosten können einerseits durch den Humankapitalansatz, der gesundheitliche Folgen auf Basis des Wertes der Produktivität eines Individuums berechnet, oder die Messung der Zahlungsbereitschaft für Nichtmarktgüter ${ }^{8}$ beziffert werden. Dass diese Berechnungen in der Praxis keineswegs unumstritten sind, zeigt die später folgende Analyse der Bewertungsverfahren von statistischen Leben in der Roll-Back-Malaria-Initiative.

Bewertungen können also monetäre Wertzuschreibungen wie Getreidepreise, Produktivitätsausfall durch gesundheitliche Folgen des Pestizideinsatzes für die Anwender oder aber auch die Zahlungsbereitschaft von Bürgern für die Vermeidung des Aussterbens einer Insektenart sein. Dabei zeigen Studien, dass auch monetäre Bewertungen Über- oder Unterschätzungen aufweisen können. So unterschieden sich bei der Schätzung des Wertes eines statistischen Lebens im Zusammenhang mit Pestizidregulierungen die Höhe des angesetzten Wertes zwischen Anwender- (35 Millionen US-Dollar) und Konsumentenleben (60.000 US-Dollar). ${ }^{9}$ Auch die Zahlungsbereitschaft von Konsumenten für die Reduzierung von Pestizidrisiken variiert stark abhängig vom Untersuchungsdesign und dem Ein- oder Ausschließen von bestimmten Risiken. ${ }^{10}$

Bewerten verweist weiterhin auf eine qualitative und sogar normative Dimension: Zunächst ist es für eine Regulierung notwendig zu bestimmen, welche Effekte bei der Entscheidungsfindung berücksichtigt werden. Pestizide können bestimmte Langzeitfolgen für Ökosysteme, die menschliche Gesundheit und landwirtschaftliche Praxis haben. Eine normative Form der Bewertung ist demnach, ob diese Faktoren in der Entscheidungsfindung berücksichtigt oder ignoriert werden. Dies scheint für manche Effekte, wie beispielsweise eine nachgewiesene Häufung von Krebserkrankungen, selbstverständlich, kann aber insbesondere bei unsicheren, indi-

8 Zahlungsbereitschaften werden auf Basis der Messung von Präferenzen erhoben. Chiara Maria Travisi, Peter Nijkamp u. Gabriella Vindigni, Pesticide Risk Valuation in Empirical Economics. A Comparative Approach, in: Ecological Economics 56, H. 4, 2006, S. 455-474.

9 Maureen L. Cropper, William N. Evans, Stephen J. Berardi, Maria M. DuclaSoares u. Paul R. Portney, The Determinants of Pesticide Regulation. A Statistical Analysis of EPA Decision Making, in: Journal of Political Economy 100, H. 1, 1992, S. 175-197.

10 Raymond J. G. M. Florax, Chiara M. Travisi u. Peter Nijkamp, A Meta-Analysis of the Willingness to Pay for Reductions in Pesticide Risk Exposure, in: European Review of Agricultural Economics 32, H. 4, 2005, S. 441-467. 
rekten oder weit in der Zukunft liegenden Folgen für Umwelt oder Gesundheit eine kritische und keinesfalls einfache Entscheidung sein.

Eine weitere Form der Bewertung kann die Einstufung eines Faktors als Ausschlusskriterium sein: Die Klassifizierung eines Pestizids als mutagen bedeutet in der EU automatisch ein Verbot der Substanz, ohne Berücksichtigung weiterer Kosten oder Nutzen. Ein Gewichten unterschiedlicher Konsequenzen wird also explizit ausgeschlossen.

Die Praktik des Gewichtens bezieht die Bewertung der einzelnen Effekte mit ein und rückt die Abwägung zwischen einzelnen Konsequenzen von Entscheidungen in einem komplexen System durch eine Quantifizierung der zugeordneten Relevanzen stärker in den Blick. ${ }^{11}$ Dies ist insbesondere deshalb wichtig, da Trade-Offs in komplexen Entscheidungsfindungen wahrscheinlich sind. In diesen Entscheidungen ist die Erreichung verschiedener Ziele unvereinbar, das heißt die Verbesserung eines Ziels ist nur mit einer einhergehenden Einschränkung anderer Ziele möglich. Dabei können Trade-Offs unterschiedlich klassifiziert werden, je nachdem ob gegenläufige Risiken (selbe oder unterschiedliche) entstehen oder andere Bevölkerungsgruppen betroffenen sind (selbe oder unterschiedliche). ${ }^{12}$ So müssen zum Beispiel bei der Zulassung von Pestiziden gesundheitliche, ökonomische und umweltbezogene Auswirkungen einbezogen und gegeneinander abgewogen werden. Die Zulassung eines neuen Pestizids kann Resistenzbildungen von Schadorganismen vorbeugen oder sicherer in der Anwendung sein, auf der anderen Seite aber bestimmte Nichtzielorganismen schädigen. ${ }^{13}$ Dabei stellt sich die Frage, welches Ziel wichtiger ist: Die Vermeidung der Resistenzbildung oder der Schutz von Nichtzielorganismen?

Insbesondere die Bestimmung von gesellschaftlich akzeptablen Wirkungen oder Grenzwerten erfordert eine Auseinandersetzung mit den Effekten der Maßnahmen auf die konkret davon betroffenen Akteure. ${ }^{14}$ Diese Aufgabe ist herausfordernd, da Effekte transnational und generationsübergrei-

11 Merriam-Webster, Definition of Evaluate, 2019: https:/www.merriam-webster.co $\mathrm{m} / \mathrm{dictionary} / \mathrm{evaluate}$ [Stand: 22.6.2019]; Merriam-Webster, Definition of Weight, 2019: https:/www.merriam-webster.com/dictionary/weight [Stand: 22.6.2019].

12 George M. Gray u. James K. Hammitt, Risk/Risk Trade-Offs in Pesticide Regulation. An Exploratory Analysis of the Public Health Effects of a Ban on Organophosphate and Carbamate Pesticides, in: Risk Analysis 20, H. 5, 2000, S. 665-680.

13 Sexton/Lei/Zilberman (2007).

14 Jeremy D. Fraiberg u. Michael J. Trebilcock, Risk Regulation. Technocratic and Democratic Tools for Regulatory Reform, in: McGill Law Journal 43, 1998, S. 835-888. 
fend sein können und häufig moralische Fragestellungen aufwerfen. Pestizide können sich in der Nahrungskette anreichern und über Ländergrenzen hinweg verbreiten. Ihre Speicherung in Böden kann Jahrzehnte überdauern und dadurch auch das Wohlergehen zukünftiger Generationen beeinflussen. Die Bewertung der weitreichenden Effekte kann damit über die Wirkung auf die gegenwärtige Gesellschaft hinausgehen. ${ }^{15}$ Damit sind in diesen Fällen weder eine klassische, wissenschaftliche Risikobewertung noch die Untersuchung gesellschaftlicher Akzeptanz als alleinige Instrumente geeignet, um komplexe Entscheidungen zu rechtfertigen. ${ }^{16}$ Die Kosten-Nutzen-Analyse bietet ein Instrument, um diese Lücke zu schließen und unterschiedlichste Folgen auf einer Argumentationsebene zu bewerten und zu gewichten. Anhand der Kosten-Nutzen-Analyse in der Pestizidzulassung soll im Folgenden aufgezeigt werden, wie sich die Evidenzpraktiken der Bewertungen und Gewichtungen aus der ökonomischen Theorie entwickelten, zunehmend formalisiert, implementiert und so zu einem festen Bestandteil evidenzbasierter Politik wurden. Dies erleichtert eine kritische Einordnung der Evidenz, die in Kosten-Nutzen-Analysen erzeugt wird.

\section{Die Etablierung der Kosten-Nutzen-Analyse in komplexen Entscheidungsprozessen}

Bei der Bewertung evidenzierter Auswirkungen spielen ökonomische Konzepte eine große Rolle. Seit der Entstehung in den 1930er Jahren in den USA entwickelte sich die Kosten-Nutzen-Analyse als eine etablierte Form der formalisierten Bewertung und Gewichtung der Auswirkungen von Regulierungen und Maßnahmen, die seither als Evidenz für Entscheidungsbegründungen eingesetzt wird. ${ }^{17}$ In der Kosten-Nutzen-Analyse gelten gute Entscheidungen als solche, bei denen der Nutzen für die Gesellschaft insgesamt größer ist, als die Kosten es sind. Folglich werden etwa gesundheitliche, ökologische und ökonomische Effekte der Zulassung eines Pestizids

15 Behnam Taebi, Bridging the Gap between Social Acceptance and Ethical Acceptability, in: Risk Analysis 37, H. 10, 2017, S. 1817-1827; Sexton/Lei/Zilberman (2007).

16 Markus Wagner, Law Talk v. Science Talk. The Languages of Law and Scence in WTO Proceedings, in: Fordham Intermational Law Journal 35, H. 1, 2016, S. 151-200.

17 Zur Geschichte der Kosten-Nutzen-Analyse vgl. Theodore M. Porter, Trust in Numbers. The Pursuit of Objectivity in Science and Public Life, Princeton 1997, S. 148-189. 
in Form von Kosten und Nutzen direkt gegenübergestellt, die vorher monetär bewertet wurden.

Für die großen Vorhaben in der New-Deal-Politik unter Roosevelt rechtfertigte der US Corps of Army die Projekte durch eine auf einem Investitionskalkül basierende Kosten-Nutzen-Berechnung. Dass diesen Berechnungen wohlfahrtsökonomische Bedeutung zugeschrieben werden konnte, zeigten die Aufsätze von Kaldor und Hicks 1939. ${ }^{18}$ Das von ihnen eingeführte Kaldor-Hicks-Kriterium einer potentiellen Paretoverbesserung ${ }^{19}$ wurde in der Folge zentraler Bestandteil von Kosten-Nutzen-Analysen. Es besagt, dass Veränderungen in der wirtschaftlichen Ordnung nicht notwendigerweise tatsächlich alle betroffenen Parteien besserstellen müssen, sondern dass es ausreicht, wenn eine solche Besserstellung von betroffenen Personen durch Umverteilung potentiell möglich ist. Durch KostenNutzen-Analysen, die das Kaldor-Hicks-Kriterium anwenden, können Eingriffe also auch dann gerechtfertigt werden, wenn die Gewinner die Verlierer für ihre Verluste potentiell kompensieren können. In diesem Fall können Verluste - zumindest theoretisch - ausgeglichen werden. Das Prinzip der potentiellen Paretoverbesserung erlaubt es also, die Praktiken des Bewertens und Gewichtens zur Gewinnung von Handlungsempfehlungen zu verwenden, selbst wenn die entsprechenden Eingriffe auch negative Konsequenzen haben. Allerdings müssen die „Gewinne“ die Verluste nur potentiell kompensieren, was mit einer Ignoranz gegenüber möglichen realen Verteilungswirkungen einhergehen kann. Überträgt man dieses theoretische Argument auf das Verbot von Pestiziden, kann also eine Reduzierung des Gesundheitsrisikos für Verbraucher (Nutzen) eine Steigerung des Ertragsverlustrisikos (Kosten) rechtfertigen: Obwohl das Verbot eine Gruppe schlechterstellt (Landwirte), bringt diese Entscheidung insgesamt einen Netto-Nutzen. ${ }^{20}$

Theoretisch basiert die Kosten-Nutzen-Analyse auf der neoklassizistischen Annahme eines vollkommenen Markts, eine Annahme, die selbst in den Wirtschaftswissenschaften höchst umstritten ist. Auch macht eine Kosten-Nutzen-Analyse eine Monetarisierung von allen Kosten und Nutzen

18 John Richard Hicks, The Foundations of Welfare Economics, in: The Economic Journal 49, H. 196, 1939, S. 696-712; Nicholas Kaldor, Welfare Propositions of Economics and Interpersonal Comparisons of Utility, in: The Economic Journal 49, H. 195, 1939, S. 549-552.

19 Nach dem Pareto-Kriterium ist eine Maßnahme dann zu befürworten, wenn die Wohlfahrt mancher Gesellschaftsmitglieder verbessert wird, ohne dass jemand schlechtergestellt wird.

20 Gray/Hammitt (2000); Cropper/Evans/Berardi/Ducla-Soares/Portney (1992). 
notwendig, ${ }^{21}$ welche eine Reihe methodischer und moralischer Fragen mit sich bringt, vor allem in der Bewertung von Effekten etwa für Umwelt oder Menschenleben, denen im Markt kein Preis zugeschrieben wird. ${ }^{22}$ Es ist also festzuhalten, dass die mit Kosten-Nutzen-Analysen erzeugte Evidenz überaus fragil ist. ${ }^{23}$ So mag auch zu erklären sein, warum sich die Anwendung von Kosten-Nutzen-Analysen in der Umweltpolitik nicht als alleiniges Instrument durchsetzen konnte: In den USA sind Kosten-NutzenAnalysen zum Beispiel im „Clean Water Act“ verboten, in der Regulierung von verschiedenen Pestiziden aber vorgeschrieben. ${ }^{24}$ Alternative Ansätze in der Umwelt- und Gesundheitspolitik bieten zum Beispiel Risikoanalysen oder die Bewertung von Risiko-Trade-Offs, in denen der Schritt der Monetarisierung nicht notwendig ist. ${ }^{25}$ Akzeptable Grenzwerte werden in diesen Verfahren nicht durch Netto-Nutzen berechnet, sondern durch naturwissenschaftlich determinierte Grenzwerte oder Bewertungen mit anderen Indikatoren, wie den qualitätskorrigierten Lebensjahren für Gesundheitseffekte. ${ }^{26}$ In diesen Verfahren fehlt aber die Möglichkeit zum direkten Vergleich, beispielsweise von Umwelteffekten und Anwenderschutz.

Bewerten und Gewichten und ihre Umsetzung im Rahmen der KostenNutzen-Analyse bedingen somit eine Vielzahl normativer Entscheidungen, um eine Wertzuschreibungen zu ermöglichen. Diese Wertzuschreibung hängt immer vom jeweiligen Kontext, z.B. von der Verteilung von Nutzen, Kosten und Risiken in der Gesellschaft, ab. Idealerweise erfolgt die Wertzuschreibung im Kontext der Gesellschaft, für die Entscheidungen zu treffen sind. Nur dann kann davon ausgegangen werden, dass die aus einer Kosten-Nutzen-Analyse resultierenden Empfehlungen die Werturteile eben dieser Gesellschaft widerspiegeln.

Ökonomen betrachten die Kosten-Nutzen-Analyse trotz ihrer Limitierungen dennoch als hilfreiches Instrument zur Rationalisierung von Regulierungsentscheidungen, die auf die Beförderung der gemeinsamen Wohl-

21 Frank B. Cross (1989) zitiert in David M. Driesen, Distributing the Costs of Environmental, Health, and Safety Protection. The Feasability Principle, Cost-Benefit Analysis, and Regulatory Reform, in: Boston College Environmental Affairs Law Review 31, H. 1, 2004.

22 Fraiberg/Trebilcock (1998).

23 Mit dieser Kritik setzen wir uns im späteren Verlauf des Kapitels noch ausführlicher auseinander.

24 Cropper/Evans/Berardi/Ducla-Soares/Portney (1992).

25 Genauere Erläuterungen zu naturwissenschaftlichen Verfahren in der Risikoanalyse sind im Kapitel Messen und Ermessen dieses Bandes zu finden.

26 Gray/Hammitt (2000). 
fahrt ${ }^{27}$ ausgerichtet sein sollen. Die Kosten-Nutzen-Analyse bietet generell eine Möglichkeit zur Systematisierung und Disziplinierung von komplexen politischen Entscheidungen. ${ }^{28}$ Sie schafft weiterhin eine Basis und gemeinsame Währung für den Austausch von Argumenten und die Abschäzung und Beurteilung zukünftig auftretender Auswirkungen. ${ }^{29}$ KostenNutzen-Kalküle werden inzwischen in unterschiedlichsten Kontexten und Staaten diskutiert und angewendet, so seit Anfang der 2000er Jahre auch in der Politik der EU. Beschleunigt wurde die Implementierung unter anderem in den 1980er und 1990er Jahren, in denen nach verschiedenen Krisen die Rolle von Wissenschaftlern und die Transparenz in Entscheidungen hinterfragt und somit die Trennung von Wissenschaft und Entscheidung forciert wurde. ${ }^{30}$ Dies hing auch zusammen mit den zunehmenden Diskursen um Risiko, Unsicherheit und Nichtwissen. Weiterhin spielte bei der Verbreitung der Kosten-Nutzen-Analyse auch die Globalisierung eine Rolle, da ein freier Handel und universal anwendbare (Entwicklungs-) programme eine vergleichbare Regulierungspraxis und damit auch Bewertungsmethoden erforderlich macht.

\section{Evidenz in der Roll-Back-Malaria-Initiative}

Globale Gesundheits- und Entwicklungsprogramme entscheiden über die Verteilung von Ressourcen nach Kosten-Nutzen-Analysen, um Auswirkungen verschiedener Programme und Investitionen zu vergleichen. Bewerten und Gewichten sind somit zentrale Praktiken dieser komplexen Strategieentscheidungen, worauf auch die Analysten selbst verweisen: „Health policymakers across the globe are facing difficult financing decisions having to balance a large unmet and rising demand for health services, costly new drugs and technologies, ambitious international guidelines, and

27 Kenneth J. Arrow, George C. Eads, Robert W. Hahn, Lester B. Lave, Roger G. Noll, Paul R. Portney, Milson Russell, Richard Schmalensee, V. Kerry Smith, u. Robert N. Stavins, Is There a Role for Benefit-Cost Analysis in Environmental, Health, and Safety Regulation?, in: Science, New Series 272, H. 5259, 1996, S. 221-22.

28 Fraiberg/Trebilcock (1998).

29 Michael P. Lynch, In Praise of Reason, Cambridge, MA 2012, S. 31.

30 Beispiele sind der Streit um die Benzol-Grenzwerte in den USA in den 1980er Jahren und der folgende Einzug der Risikoanalyse mit einer Trennung von Risikobewertung und -management in der Regulierungspraxis. 
severely constrained health budgets. “31 Insbesondere bei Infektionskrankheiten wie Malaria oder HIV im Globalen Süden, die einerseits massive wirtschaftliche Folgen haben und denen man andererseits oftmals in einem völlig unterfinanzierten und strukturell zerrütteten Gesundheitssystem begegnet, spielen nicht nur die erwarteten Investitionskosten, sondern auch die Abschätzung und Miteinrechnung der langfristigen finanziellen Konsequenzen eine entscheidende Rolle. ${ }^{32}$

Wie ökonomisierte Entscheidungsprozesse und insbesondere die Kosten Nutzen-Analysen in der internationalen Gesundheitspolitik zum Tragen kommen, lässt sich deshalb gut mit Beispielen der globalen Roll-Back-Malaria-Initiative (RBM) illustrieren. ${ }^{33}$ Dieses 1998 gegründete Netzwerk unter Federführung der Weltgesundheitsorganisation (WHO) versucht, die Anstrengungen von Organisationen der Vereinten Nationen, von NGOs, Stiftungen, Forschungs- und Entwicklungshilfeprogrammen sowie privatwirtschaftlichen und lokalen Initiativen im Umfeld der Malaria-Bekämpfung zu koordinieren und mit Ressourcen auszustatten. ${ }^{34}$ Hauptaufgabe der Roll-Back-Malaria-Zentrale in Genf ist es, Konsens über umsetzbare Strategien zwischen den über 500 beteiligten Organisationen herzustellen. Sie steht damit nicht nur vor der Herausforderung, eine globale Strategie zu entwickeln, die sich in diversen lokalen Kontexten, insbesondere in verarmten Regionen des südlichen Afrikas sowie Südostasiens, bewährt. Ziel von Roll-Back-Malaria ist ebenfalls, diese Strategieentscheidungen zwischen den Beteiligten zu kommunizieren und zu vermitteln. Neben einer

31 Michelle Remme, Melisa Martinez-Alvarez u. Anna Vassall, Cost-Effectiveness Thresholds in Global Health. Taking a Multisectoral Perspective, in: Value in Health. The Journal of the International Society for Pharmacoeconomics and Outcomes Research 20, H. 4, 2017, S. 699-704, hier S. 699.

32 Vgl. bspw. Chelsea Clinton u. Devi Lalita Sridhar, Governing Global Health. Who Runs the World and Why?, New York, NY 2017, insbesondere das Kapitel Twentyfirst-Century Governance, S. 119-160; Felix Stein u. Devi Sridhar, The Financialisation of Global Health, in: Wellcome Open Research 3, 2018, S. 17.

$33 \mathrm{Zu}$ RBM als evidence based policy s. Malaria Advocacy Working Group, Evidence for Advocacy. Key Statistics on the Fight Against Malaria. Roll Back Malaria Partnership, Genf 2015; Richard W. Steketee u. Carlos C. Campbell, Impact of National Malaria Control Scale-up Programmes in Africa. Magnitude and Attribution of Effects, in: Malaria Journal 9, 2010, S. 299; Randall A. Kramer et al., Using Decision Analysis to Improve Malaria Control Policy Making, in: Health Policy 92, H. 2-3, 2009, S. 133-140.

34 David N. Nabarro u. Elizabeth M. Tayler, The "Roll Back Malaria" Campaign, in: Science 280, H. 5372, 1998, S. 2067-2068; Nicole Fraser, Partnerships for Malaria Control. Engaging the Formal and Informal Private Sectors; a Review Commissioned by the UNICEF/UNDP/World Bank/WHO, Genf 2006. 
Vielzahl von beteiligten Gesundheits- und Entwicklungshilfe-Organisationen müssen die betroffenen Länder und Regierungen von internationalen Strategien und gemeinsamen Zielvereinbarungen ebenso überzeugt werden wie die Geldgeber der Initiativen, die staatliche wie auch privatwirtschaftliche Stellen umfassen. ${ }^{35}$

Entscheidungen müssen folglich in einem komplexen System getroffen werden, das auf international wie lokal tätige Akteure und ihre verschiedensten professionellen Hintergründe, Ressourcen, Motive und Interessen ausgerichtet ist. Wie im breiteren Feld der globalen Entwicklungs- und Gesundheitspolitik sind auch in der Roll-Back-Malaria Partnership Strategievorgaben durch formalisierte Entscheidungsprozesse zu verzeichnen. Standardindikatoren und vergleichbare Bewertungsmaßstäbe dienen hier der Transparenz und Nachvollziehbarkeit von Zielvereinbarungen über fachliche und lokale Grenzen hinweg. ${ }^{36}$ Gleichzeitig bringt die Vielzahl der beteiligten Institutionen und Akteure einen gewissen Zwang zur Standardisierung und Ökonomisierung von Entscheidungsprozessen mit sich. Dieses Vorgehen hat erstens zur Folge, dass die möglichen Konsequenzen von Roll-Back-Malaria-Initiativen stets vom lokalen Kontext abstrahiert und kontextübergreifend systematisiert werden. Es werden also beispielsweise Infektions- oder Sterberaten in bestimmten Altersgruppen verglichen und mit der Einführung von bestimmten gesundheitspolitischen Maßnahmen ins Verhältnis gesetzt. ${ }^{37}$ Zweitens bedeutet es, dass - im Sinne einer Kos-

35 S. bspw. RBM Partnership to End Malaria, RBM Partnership Strategic Plan 20182020, 2018, S. 13: https://endmalaria.org/sites/default/files/RBM-Strategic-Plan-dig ital-JW-220218.pdf [Stand: 22.6.2019]; Zur Entwicklung der Strategien der RBM s. Clinton/Sridhar (2017), S. 14-16.

36 Vgl. J. H. Remme, F. Binka u. David N. Nabarro, Toward a Framework and Indicators for Monitoring Roll Back Malaria, in: The American Journal of Tropical Medicine and Hygiene 64, 1-2 Suppl., 2001, S. 76-84. Bspw. Percentage reduction in mortality of children $<5$ years old, Percentage reduction in malaria incidence rate, Percentage of districts systematically using health information for planning etc. Für eine Liste von Standardindikatoren s. Tabelle 1.

37 S. bspw. RBM Partnership to End Malaria, Annual Report 2018, S. 17-20: https:// endmalaria.org/sites/default/files/RBM\%20Annual\%20Report\%202018_EN.pdf [Stand: 22.6.2019]; Roll Back Malaria Partnership, Evidence for Advocacy. Key Statitics on the Fight for Malaria, 2015: http://www.makingmalariahistory.org/wp -content/uploads/2015/06/Malaria_Evidence-for-Advocacy_April_2015.pdf [Stand: 22.6.2019]; RBM Partnership to End Malaria, Annual Report 2017, S. 8 u. 11: https://endmalaria.org/sites/default/files/RBM\%20Annual\%20Report_web.pdf [Stand: 22.6.2019]; Roll Back Malaria Partnership, Multisectoral Action Framework for Malaria, 2015, S. 15-29: https://endmalaria.org/sites/default/files/9_Multi sectoral-Action-Framework-for-Malaria.pdf [Stand: 22.6.2019]. 
ten-Nutzen-Analyse - sowohl die aufzuwendenden Ressourcen wie auch die Effekte der Roll-Back-Malaria-Maßnahmen monetär bewertet werden. Wie bereits erläutert, zeigt sich auch hier das Problem des Einbezugs von nicht monetären Gütern, wie zum Beispiel der Gesundheit von Menschen, sowie der Abschätzung von unsicheren oder indirekten Langzeitfolgen beispielsweise durch Insektizideinsätze im Rahmen der Malariamückenbekämpfung. ${ }^{38}$

Ein weiteres Problem evidenzbasierter Politik im Globalen Süden ist, dass sie mit Parametern und Determinanten operiert, die für deutlich reichere Länder entwickelt wurden. ${ }^{39}$ Die Ökonomen Lisa Robinson, James Hammitt und Lucy O'Keeffe haben dies am Beispiel der VSL-Schätzungen (Value per Statistical Life / Value of Mortality Risk Reductions) erläutert, die breit für Entwicklungsprogramme eingesetzt werden, sich aber ursprünglich auf die US-amerikanische Wirtschaft und Gesellschaft bezogen beziehungsweise für OECD-Mitgliedsstaaten entwickelt wurden. Allein die deutlich höhere Säuglingssterblichkeit, aber auch die generell geringere Lebenserwartung in Entwicklungsländern stellen Herausforderungen an die ökonomischen Analysen dar, denen in der Regel aber gar nicht begegnet wird. Robinson und Koautoren bilanzieren beispielsweise bezogen auf die Value-per-Statistical-Life-Bewertung von Kindern und Erwachsenen, dass diese sich nach reichen Ländern richtet, weil dies die einzige Forschungsgrundlage ist. „For low- and middle-income countries, little empirical research is available and it is unclear whether the same patterns hold. "40 Dieser von Robinson und anderen kritisierte Mangel an Forschung ist

38 Catherine A. Goodman, Paul G. Coleman u. Anne J. Mills, Cost-Effectiveness of Malaria Control in Sub-Saharan Africa, in: The Lancet 354, H. 9176, 1999, S. 378 385; Raphael Mendonça Guimarães, Carmen Ildes Rodrigues Fróes Asmus u. Armando Meyer, DDT Reintroduction for Malaria Control. The Cost-Benefit Debate for Public Health, in: Cadernos de saude publica 23, H. 12, 2007, S. 28352844. Zur Bewertung von Umweltfolgen der DDT-Einsätze s. stellvertretend William P. Kabasenche u. Michael K. Skinner, DDT, Epigenetic Harm, and Transgenerational Environmental Justice, in: Environmental Health. A Global Access Science Source 13, 2014, S. 62.

39 Eine der VSL-Schätzungen vergleichbare Kritik existiert für das Konzept der DALYs (Disability-Adjusted Life Years bzw. Disease-Adjusted Life Years) in globalen Gesundheitsprogrammen. Rachel Parks, The Rise, Critique and Persistence of the DALY in Global Health, in: The Journal of Global Health, 2014: https:/www.ghj ournal.org/the-rise-critique-and-persistence-of-the-daly-in-global-health/ [Stand: 21.8.2019].

40 Lisa A. Robinson, James K. Hammitt u. Lucy O'Keeffe, Valuing Mortality Risk Reductions in Global Benefit-Cost Analysis, in: Journal of Benefit-Cost Analysis 10, S1, 2019, S. 15-50, hier S. 34. 
aber für Kollaborationen wie Roll-Back-Malaria kein Grund, von Value-perStatistical-Life-Schätzungen abzusehen und vorerst weitere, kontextbezogene Forschungen abzuwarten. Vielmehr ist in den Publikationen der RollBack-Malaria-Partnership von der Diskrepanz zwischen Entwicklungs- und Anwendungskontext oder einem folglichen Mangel an Evidenz keine Rede. $\mathrm{Zu}$ sehr steht dazu die Evidenzbasiertheit der Kampagnen im Vordergrund. ${ }^{41}$

In den Publikationen der Roll-Back-Malaria-Partnership sind Strategien und Zielvereinbarungen stets sehr allgemein formuliert, da sie auf internationales Publikum und in der Regel auch auf eine globale Anwendbarkeit ausgerichtet sind. Gleichzeitig spielen Verweise auf fortlaufende Evaluierung der eigenen Arbeit und Schlussfolgerungen aus dem Geleisteten eine entscheidende Rolle. ${ }^{42}$ Die Publikationen leben von einer Mischung aus Slogans wie beispielsweise „Vision: A world free from the burden of Malaria" und sachlich aufbereiteten Textinformationen sowie Bildern, die Assoziationen bedienen sollen. Sie formulieren ihre Strategien kurz und einprägsam und kombinieren emotionalisierende Fotos mit Statistiken und Diagrammen. Insbesondere Fotos lächelnder schwarzer Kinder zeigen dem Leser, dass es hier um mehr geht als bloße Finanzkalkulationen, nämlich um die Entwicklung und um die Zukunft des afrikanischen Kontinents. ${ }^{43}$ Dass ökonomische Analysen allgemeinpolitisch eingebettet und mit moralischen Werten versehen werden, findet sich auch in der Roll-Back-Malaria zugrundeliegenden Forschungsliteratur. „The compelling economic case for fighting malaria underscores the social and ethical merits of eradicating this disease", schreibt beispielsweise die Forschungsgruppe um den Ökonomen Marc Purdy. „We hope that greater awareness of the economic case for eradication will better serve the humanitarian imperative of rid-

41 Vgl. Special Issue: Lisa A. Robinson, James K. Hammitt, Dean T. Jamison u. Damian G. Walker, Conducting Benefit-Cost Analysis in Low- and Middle-Income Countries. Introduction to the Special Issue, in: Journal of Benefit-Cost Analysis, S1, 2019, S. 1-14; Gavin Yamey, Global Campaign to Eradicate Malaria. Roll Back Malaria Has Achieved a High Profile But Little Real Action, in: British Medical Journal 322, 2001, S. 1191f.

42 Bspw. RBM Partnership to End Malaria (2018); RBM Partnership To End Malaria (2017), S. 3; Roll Back Malaria Partnership, Action and Investment to Defeat Malaria 2016-2030, 2015: https://endmalaria.org/sites/default/files/RBM_AIM_Re port_0.pdf [Stand: 22.6.2019].

43 Siehe die Jahresberichte, bspw. RBM Partnership to End Malaria (2018); RBM Partnership To End Malaria (2017). 
ding the world of malaria." 44 Dieses Changieren zwischen zweckrationalen bzw. ökonomischen Gründen und dem Bezug auf humanitäre Ziele ist auch für die Frage nach Evidenzpraktiken interessant: Auf die politisch oder moralisch geframte Frage nach dem Umgang mit sozialer Ungleichheit und unterschiedlichen Lebenschancen liefern hier ökonomische Berechnungen die Evidenz.

Wie groß dabei die Diskrepanz zwischen globaler Plakativität und konkreter Evidenz sein kann, zeigt beispielsweise eine der wichtigsten RollBack-Malaria-Strategien der letzten Jahre: die Einführung von mit Insektiziden behandelten Netzen (ITNs/LLITNs: Insecticide-Treated Nets / Long Lasting Insecticide-Treated Nets) zum Schutz vor dem Vektor der Krankheit, der Anopheles-Mücke. Diese Netze gehören seit den 1990er Jahren zum Instrumentarium der weltweiten Malariakontrolle. Dank ihrer ressourcenschonenden Anforderungen entwickelten sie sich rasch zu einer der Schlüsseltechnologien, die symbolisch für die Prinzipien globaler Gesundheitspolitik stehen: günstig in der Produktion, simpel in der Anwendung, global einsetzbar. ${ }^{45}$ So vermerkt der Roll-Back-Malaria-Jahresbericht von 2017 die Verteilung von 582 Millionen Insecticide-Treated Nets zwischen 2014 und 2016 und unterstreicht den effizienten Charakter dieser Intervention. ${ }^{46}$

Betrachtet man jedoch die Anfangsgeschichte und die fortschreitende Einführung der Netze, zeichnet sich dies keineswegs durch transparente und nachvollziehbare Bewertungs- und Gewichtungsprozesse aus. ${ }^{47}$ Insektizidnetze wurden zunächst in den 1990er Jahren mit randomisierten kon-

44 Mark Purdy, Matthew Robinson, Kuangyi Wei u. David Rublin, The Economic Case for Combating Malaria, in: The American Journal of Tropical Medicine and Hygiene 89, H. 5, 2013, S. 819-823.

45 James L. A. Webb, The Long Struggle Against Malaria in Tropical Africa, Cambridge 2014, S. 143-151; Jenny Hill, Jo Lines u. Mark Rowland, Insecticide-Treated Nets, in: D. H. Molyneux (Hg.), Control of Human Parasitic Diseases, New York 2007, S. 77-128; Carrie B. Dolan, Ariel BenYishay, Karen A. Grépin, Jeffery C. Tanner, April D. Kimmel, David C. Wheeler u. Gordon C. McCord, The Impact of an Insecticide Treated Bednet Campaign on All-Cause Child Mortality. A Geospatial Impact Evaluation from the Democratic Republic of Congo, in: PLOS ONE 14, H. 2, 2019: https://doi.org/10.1371/journal.pone.0212890.

46 RBM Partnership To End Malaria (2017), S. 8.

47 Zur Diskussion von ITNs: William Brieger, Africa Health. The Challenge of Using and Misusing Insecticide-Treated Bed Nets, in: Africa Health 39, 2017, S. 1315; Robert K. Peterson, Loren M. Barber u. Jerome J. Schleier, Net Risk. A Risk Assessment of Long-Lasting Insecticide Bed Nets Used for Malaria Management, in: The American Journal of Tropical Medicine and Hygiene 84, H. 6, 2011, S. 951-956. 
trollierten Studien (RCTs) in Siaya in Westkenia erprobt. Die Historikerin Kirsten Moore-Sheeley beschreibt, wie Forscher der U.S. Centers for Disease Control und des Kenya Medical Research Institute kontinuierlich ihre Forschungsfragen und -praktiken der lokalen Bevölkerung und den Gegebenheiten anpassten, diese lokale Spezifik aber nicht in ihren Schlussfolgerungen erwähnten, geschweige denn die Reichweite ihrer Ergebnisse entsprechend eingrenzten. ${ }^{48}$ In Anbetracht dieses Entstehungskontextes überrascht es nicht, dass die Einführung der Insektizidnetze dieselbe Kritik trifft, die auch gegenüber weltweiter Gesundheitspolitik im Allgemeinen geäußert wird: Globale Gesundheitstechnologien und -politiken ignorieren vielfach lokale Spezifika. Sie versäumen es, lokales Wissen zu nutzen und sie versuchen, Technologien zu implementieren, die für andere Kontexte entwickelt wurden und nicht ubiquitär funktionieren. ${ }^{49}$

Folglich zeigt sich auch in der lokalen Implementierung der Roll-BackMalaria-Strategien, dass den formalisierten Kriterien der internationalen Analysen hier weniger Relevanz zukommt und sie keineswegs gleichmäßig angewandt werden. Beispielsweise haben der Zugang zu Medikamenten und weiteren Ressourcen sowie politische Machtverhältnisse und kulturelle Prägungen entscheidenden Einfluss auf den Erfolg internationaler Vorgaben. ${ }^{50}$ Im Versuch, lokale Spezifika zu berücksichtigen und bestimmte Zielgruppen zu erreichen, wenden lokale Akteure die Vorgaben aus Genf nicht nur äußerst freihändig an, sondern definieren auch eigene Kriterien für Erfolg und Misserfolg. ${ }^{51}$ Diese Diskrepanz zwischen lokalen und inter-

48 Kirsten Moore-Sheeley, Nothing but Nets. The History of Insecticide-Treated Nets in Africa, 1980s-Present. Dissertation Johns Hopkins University 2017. Zur Geschichte der ITNs s. außerdem Paul J. Krezanoski, Delivering Insecticide-Treated Nets for Malaria Prevention. Innovative Strategies, in: Research and Reports in Tropical Medicine 7, 2016, S. 39-47.

49 Vgl. Vincanne Adams, Metrics. What Counts in Global Health, Durham, NC 2016. Bocar Kouyaté, Ali Sie, Maurice Yé, Manuela de Allegri u. Olaf Müller, The Great Failure of Malaria Control in Africa. A District Perspective from Burkina Faso, in: PLOS Medicine 4, H. 6, 2007, e127; Amy Barnes u. Justin Parkhurst, Can Global Health Policy be Depoliticized? A Critique of Global Calls for EvidenceBased Policy, in: Garrett Wallace Brown, Gavin Yamey u. Sarah P. Wamala (Hg.), The Handbook of Global Health Policy, Bd. 84, Chichester, West Sussex u. Malden, MA 2014, S. 157-173.

50 Yamey (2001); James Pfeiffer u. Mark Nichter, What Can Critical Medical Anthropology Contribute to Global Health?, in: Medical Anthropology Quarterly 22, H. 4, 2008, S. 410-415.

51 S. bspw. Awash Teklehaimanot u. Paola Mejia, Malaria and Poverty, in: Annals of the New York Academy of Sciences 1136, 2008, S. 32-37; Webb (2014), S. 168 172. 
nationalen Bewertungskriterien unterläuft jedoch nicht nur die versprochene Transparenz der Roll-Back-Malaria-Initiative, sondern geht vielfach auch mit einer auf internationaler Ebene geringen Aufmerksamkeit für die tatsächlichen lokalen Ergebnisse und Möglichkeiten einher. In dieser Hinsicht lassen sich die Grenzen evidenzbasierter globaler Politik durchaus mit der im Kapitel De- und Re-Kontextualisieren referierten Kritik an evidenzbasierter Medizin vergleichen: Auch hier geht das Streben nach Evidenz und Vergleichbarkeit im Zweifel zu Lasten der im Einzelfall erzielten Ergebnisse. ${ }^{52}$

Das Problem der Roll-Back-Malaria-Initiative ist in dieser Hinsicht also nicht in erster Linie der grundsätzliche Mangel an Evidenz, sondern die fehlende Berücksichtigung ihrer Spezifik und deren Bewertung und Gewichtung. Bei der Einführung von Insecticide-Treated Nets gab es nach jahrelangen Experimenten durchaus Evidenz für ihre Wirksamkeit, nur war diese eben kontextgebunden. Diese Einschränkung ging jedoch in den ökonomisierten Entscheidungsprozessen unter. Am Beispiel der InsecticideTreated Nets wird deutlich, dass evidenzbasierte Gesundheitspolitik nicht automatisch mit transparenten Entscheidungsprozessen einhergehen muss. Vielmehr wird, wenn die Gewichtung spezifischer Evidenz nicht offengelegt wird, damit der gesamte Entscheidungsprozess verschleiert. Bewertungskriterien für erfolgreiche Roll-Back-Malaria-Maßnahmen müssten also viel stärker auf ihre Spezifik befragt werden und diese Spezifik sollte in die Bewertung und Gewichtung von Forschungsbefunden und Ergebnissen einbezogen werden.

Zweitens illustrieren Analysen im Zuge der Roll-Back-Malaria-Initiative ein weiteres generelles Problem evidenzbasierter Entscheidungsprozesse in verarmten Regionen der Welt: Diese Analysen basieren auf Parametern, die für einen anderen Kontext entwickelt wurden, nämlich für die reicheren Länder des Nordens. Versuche, Forschungen voranzutreiben, die Determinanten für Low Income / Lower Middle Income Countries entwickeln, stecken dagegen noch in den Anfängen. Hier zeigt sich also, was passieren kann, wenn die Forderung nach evidenzbasierter Politik auf eine Forschungslücke trifft: Anstatt diese Lücke zu markieren, wird der Forderung nach Evidenz nachgekommen. Inwieweit diese Evidenz aber zu den Anwendungskontexten passt, ist kaum nachvollziehbar.

Dieser Befund knüpft an die breitere These einer Ignoranz der (Entwicklungs-)Ökonomen gegenüber der Realität des Globalen Südens an, obwohl diese Experten zu genau diesen Regionen forschen und damit po-

52 S. Kapitel De-und Re-Kontextualisieren in diesem Band. 
litische Entscheidungen beeinflussen. Abhijit Banerjee und Esther Duflo, die Autoren des des Grundsatzwerks Poor Economics, sehen darin einen entscheidenden Grund für die Ineffizienz der Entwicklungshilfe.

Wenn wir das träge, schematische Denken aufgeben, das jedes Problem auf die gleichen allgemeinen Prinzipien reduziert, wenn wir den Armen richtig zuhören und uns bemühen, die Logik ihrer Entscheidungen zu verstehen, wenn wir akzeptieren, dass wir uns irren können, und jede scheinbar noch so vernünftige Idee empirischen Tests unterziehen, dann werden wir nicht nur in der Lage sein, effektive Maßnahmen zu entwickeln, sondern auch besser verstehen, warum die Armen so leben, wie sie leben. ${ }^{53}$

Liest man diesen Aufruf im Lichte unserer Überlegungen zu Evidenzpraktiken als Aushandlungsprozesse um die Gültigkeit von Wissen, wird klar, dass er nicht nur auf eine andere Politik, sondern insbesondere auf andere Modi des Überzeugens abzielt. Die Parameter, nach welchen wir Strategien der Entwicklungspolitik beurteilen, nach denen Expertise uns glaubwürdig scheint und Wissen als gesichert gilt, sind zu hinterfragen.

\section{Evidenz als Entscheidungshilfe in der Kritik}

Die Kosten-Nutzen-Analyse soll vielen Ansprüchen gerecht werden. Eine differenzierte Betrachtung von Kontroversen ermöglicht eine kritische Einordnung ihrer Ergebnisse und der Evidenzpraktiken Bewerten und Gewichten im Allgemeinen. Das Beispiel der Roll-Back-Malaria-Strategien illustriert vorrangig spezifische Probleme evidenzbasierter Politik im Globalen Süden. Die Bewertung und Gewichtung von Evidenz im Rahmen der Kosten-Nutzen-Analysen öffnet jedoch auch ein allgemeineres, fundamentaleres Feld für Kontroversen. Einige dieser Kontroversen sollen im Folgenden diskutiert werden. Sie entstehen aus mehreren Faktoren, die bereits im bisherigen Verlauf des Kapitels aufgezeigt wurden: Zunächst ist die KostenNutzen-Analyse an einem sehr kritischen Punkt im Prozess der Konfliktbewältigung angesiedelt, weiterhin sind an ihre Ergebnisse weitreichende Entscheidungen geknüpft. Oft finden sie in komplexen Problemfeldern Anwendung, in denen eine Entscheidung über „akzeptabel“ oder „inakzeptabel“ keineswegs einfach möglich ist. Auch ist zu bedenken, dass sich

53 Abhijit V. Banerjee u. Esther Duflo, Poor Economics. Plädoyer für ein neues Verständnis von Armut, München 2012, S. 351f. 
Kosten-Nutzen-Analysen in einem westlichen und neoklassizistischen Kontext entwickelten, dessen Annahmen nicht problemlos auf andere Kontexte übertragen werden können.

Die erste Kontroverse entsteht aus der kritischen Verortung der KostenNutzen-Analyse und bezieht sich auf die Verwissenschaftlichung von Diskursen und deren Auswirkungen auf demokratische Prozesse. Kosten-Nutzen-Analysen zielen eigentlich darauf ab, die wertenden Elemente im Prozess der evidenzbasierten Politik (vor allem im Hinblick auf effiziente Ressourcennutzung) zu „rationalisieren“. Gleichzeitig trägt diese Entwicklung aber auch zu einer Verwissenschaftlichung und Entrückung des Bewertungsprozesses bei, was eine Beteiligung der Öffentlichkeit erschwert. Die Voraussetzung technischer und wissenschaftlicher Analysen für eine Bewertung komplexer Situationen schreibt Experten automatisch eine hohe politische Autorität zu und erschwert Laien den Zugang zum Prozess, da sie nicht über das notwendige Wissen und/oder die entsprechenden Ressourcen verfügen. ${ }^{54}$ Für ein Verständnis des Zulassungsprozesses von Pestiziden (und damit die Möglichkeit zur Kritik an diesem) ist zum Beispiel umfangreiches naturwissenschaftliches Wissen aus der Toxikologie oder Ökotoxikologie notwendig. Diese Verwissenschaftlichung kann zu Anpassungsstrategien anderer Akteure führen, die ihrerseits ebenfalls (wissenschaftlich basierte) Instrumente und Strategien entwickeln, um sich am technischen Prozess zu beteiligen. Kinchy konnte zum Beispiel in einer Studie zum Streit um die Zulassung von gentechnisch veränderten Maissorten nachweisen, dass Interessengruppen selbst begannen, wissenschaftliches Wissen zu nutzen und zu produzieren. ${ }^{55}$ Dies kann wiederum als problematisch angesehen werden, da sich hier besonders starke oder durch Geld unterstützte Gruppen durchsetzen können, die die Präferenzen der Gesellschaft möglicherweise verzerrt abbilden. ${ }^{56}$ Somit kann die KostenNutzen-Analyse entweder als antidemokratisches oder als demokratiestützendes Instrument gesehen werden: Einerseits schließt sie durch Technisierung Laien aus und birgt die Gefahr der Verzerrung, schützt aber Entscheider und Bürger vor dem Einfluss von Lobbyismus, Ideologie oder öffentlicher Hysterie. ${ }^{57}$

54 Abby J. Kinchy, Anti-Genetic Engineering Activism and Scientized Politics in the Case of "Contaminated" Mexican Maize, in: Agriculture and Human Values 27, H. 4, 2010, S. 505-517; Cass R. Sunstein, The Cost-Benefit Revolution, Cambridge 2018 .

55 Kinchy (2010), S. 505-517.

56 Sunstein (2018).

57 Ebd.; Driesen (2004); Dobrow/Goel/Upshur (1998). 
Die zweite Kontroverse, die hier thematisiert werden soll, entsteht aus der Komplexität der Problemfelder und bezieht sich auf das Framing- und Wissensproblem der Kosten-Nutzen-Analysen. In dieser Kontroverse finden sich Argumente um die Methodik der Kosten-Nutzen-Analyse selbst. Kosten-Nutzen-Analysen funktionieren an sich als eine Art von Framing, das alternative Arten der Bewertung von Konsequenzen ausschließt. ${ }^{58}$ In diesen Analysen ist außerdem selten universell definiert, welche Arten von Kosten und Nutzen eingeschlossen werden. ${ }^{59}$ Dadurch ist ein Framing, also der Ein- und Ausschluss von Effekten, abhängig von den durchführenden Experten. Effekte können durch Ignoranz externalisiert werden und finden dadurch keinen Eingang in den Entscheidungsprozess.

Diese Kritik ist übertragbar aus dem Diskurs Realismus - Konstruktivismus in der Risikoanalyse. Während im Realismus davon ausgegangen wird, dass die wissenschaftliche Bewertung die objektive Realität am besten abbildet, argumentieren Konstruktivisten, dass das Framing der Risikoanalyse nur Konventionen einer bestimmten Elite wiedergibt, indem eine Gruppe einen für die Gruppe logischen Konzeptrahmen bestimmt. ${ }^{60}$ Vergleichbar argumentiert der Ökonom Friedrich von Hayek 1974 in seiner Analyse Anmaßung von Wissen, dass die „Planer" nie über das gesamte, relevante Wissen aller Akteure verfügen können. ${ }^{61}$ Dies hat, so schreibt Sunstein, weitreichende Folgen für die Rechtfertigung von Kosten-Nutzen-Analysen: „If cost-benefit analysis is essential to sensible judgements, incomplete knowledge, when it exists, would appear to be a serious and potentially devastating problem.“62 Dass unvollständige Wissensbestände aber häufig vorkommen, ohne dass daraus Konsequenzen für globale Politik gezogen werden, hat das Beispiel der Roll-Back-Malaria-Analysen gezeigt. Auch in der Zulassung von Pestiziden macht die Komplexität der Folgen für Gesellschaft und Umwelt vollständiges Wissen zu einer Utopie: Wie bereits erläutert, ziehen Entscheidungen unterschiedlichste Effekte

58 Saltelli/Giampietro (2017).

59 Driesen (2004).

60 Andreas Klinke u. Ortwin Renn, A New Approach to Risk Evaluation and Management. Risk-Based, Precaution-Based, and Discourse-Based Strategies, in: Risk Analysis 22, H. 6, 2002, S. 1071-1094.

61 Hayek geht davon aus, dass sich dies durch Marktautomatismen lösen lässt. Dagegen sprechen Konstruktivisten im Risikodiskurs eher von Beteiligung der Gesellschaft. Friedrich August von Hayek, Die Anmaßung von Wissen. Neue Freiburger Studien, Tübingen 1996.

62 Sunstein (2018), S. 80. 
auf lokaler und globaler Ebene nach sich, meist verknüpft mit hoher Unsicherheit. ${ }^{63}$

Weiterhin bemängeln Kritiker von Kosten-Nutzen-Analysen, dass häufig die Verteilung von Nutzen und Kosten nicht ausreichend berücksichtigt wird. ${ }^{64}$ Dass dies in der Pestizidregulierung ein relevantes Kriterium ist, konnten die Agrarökonomen Lichtenberg, Parker und Zilberman bereits 1988 zeigen: Sie wiesen nach, dass sich die Wohlfahrtseffekte für verschiedene Akteure abhängig von den Angebots- und Nachfragereaktionen deutlich unterschieden. Potentielle Risiko-Trade-Offs, welche im vorangehenden Verlauf des Kapitels erläutert wurden, werden somit vernachlässigt oder verschleiert. Ein weiterer Kritikpunkt ist, dass die Festlegung einer Rate zur Diskontierung von zukünftigen Risiken und Nutzen, z.B. bei generationsübergreifenden Effekten, schwierig ist und oft vernachlässigt wird. Auch Substitutions- und Mitanahmeeffekte sind Faktoren, die relevant sind, aber selten berücksichtigt werden..$^{65}$

Methodische Probleme betreffen insbesondere die Möglichkeit der Messung von Wohlfahrtseffekten im Allgemeinen; ${ }^{66}$ und das Problem der Abbildung von Nutzen durch vermiedene Schäden im Besonderen. Bei der Berechnung des Nutzens von Pestiziden wird beispielsweise oft vernachlässigt, dass sie Qualitätsverluste durch Schadorganismen am Getreide verhindern können, obwohl diese ca. 20 Prozent des durch Pestizideinsatz erzielten Mehrumsatzes ausmachen. Verschiedene Studien zeigen, dass die Vernachlässigung der Qualitätseffekte zu einer Unterschätzung der Wohlfahrtseffekte führt. ${ }^{67}$ Weiterhin können die Messungen von Wohlfahrtseffekten, wie alle wissenschaftliche Studien, widersprüchliche Ergebnisse produzieren. So zeigen verschiedene Studien zum Einfluss vom Rückstandshöchstgehalten von Pestiziden auf den Handel mit den regulierten Lebensmitteln positive oder negative Effekte. ${ }^{68}$

63 Sexton/Lei/Zilberman (2007).

64 Fraiberg/Trebilcock (1998); Sunstein (2018)

65 Fraiberg/Trebilcock (1998); Arrow et al. (1996).

66 Eine Kosten-Nutzen-Analyse kann im schlechtesten Fall entkoppelt von Wohlfahrtseffekten sein, da sie nur ein Proxy für Wohlfahrt ist; Sunstein (2018).

67 Kentaro Kawasaki u. Erik Lichtenberg, Quality versus Quantity Effects of Pesticides. Joint Estimation of Quality Grade and Crop Yield, in: AAEA Annual Meeting, San Francisco 2015; Bruce A. Babcock, Erik Lichtenberg u. David Zilberman, Impact of Damage Control and Quality of Output. Estimating Pest Control Effectiveness, in: American Journal of Agricultural Economics 74, 1992, S. 163-172.

68 Caroline E. Handford, Christopher T. Elliott u. K. Campbell, A Review of the Global Pesticide Legislation and the Scale of Challenge in Reaching the Global 
Die Messung von Wohlfahrtseffekten basiert weiterhin in vielen Fällen auf der vorangestellten Risikobewertung: Gesundheitseffekte von Pestiziden können erst dann monetär bewertet werden, wenn sie naturwissenschaftlich identifiziert und quantifiziert wurden. Erst wenn leberschädigende Eigenschaften eines Pestizids beziffert wurden, kann dieser Effekt monetär bewertet werden. Die Kosten-Nutzen-Analyse ist dadurch stark abhängig von der quantitativen Risikobewertung und übernimmt deren Problematiken, zum Beispiel in der Extrapolation von Effekten oder Datenlücken und den damit verbundenen Unsicherheiten, welche auch im Kapitel Messen und Ermessen dieses Bandes diskutiert werden. ${ }^{69}$ Zusammengefasst können Kosten-Nutzen-Analysen ein Framing- oder Wissensproblem haben, da sie sehr komplex sind, sehr viele Daten benötigen und auf vielen Annahmen und vorausgegangenen Verfahren beruhen. ${ }^{70}$

Eine weitere Kontroverse im Kontext von Kosten-Nutzen-Analysen betrifft moralische Fragestellungen. Dazu gehört zunächst die implizierte Notwendigkeit der Bewertung und Quantifizierung von menschlichem Leben oder anderen nicht monetären Effekten. Dies macht fragwürdige Annahmen über den Wert des menschlichen Lebens notwendig, für die es bisher keine unumstrittene Methode gibt, ${ }^{71}$ auch wenn dieses Problem in der Wissenschaft bereits seit Starr (1969) in verschiedensten Ansätzen behandelt wird. ${ }^{72}$ Dies wurde bereits am Beispiel der Pestizide und der RollBack-Malaria-Initiative im vorangegangenen Verlauf des Kapitels deutlich: Zum einen können unterschiedliche Wertzuschreibungen für die Leben von verschiedenen Akteuren kritisch betrachtet werden (Konsumenten vs. Anwender). Zum anderen kann die Übertragung des Value per Statistical Life in unterschiedliche Kontexte problematisch sein. ${ }^{73}$ Ähnlich verhält es sich mit der Bewertung von anderen nicht monetären Konsequenzen wie zum Beispiel Effekten auf Biodiversität oder der Bewertung von Zufriedenheit oder moralischen Bedenken, deren direkte Wohlfahrtseffekte für eine Gesellschaft oft schwierig zu erkennen und quantifizieren sind. ${ }^{74}$ Auch

Harmonization of Food Safety Standards, in: Integrated Environmental Assessment and Managment 11, H. 4, 2015, S. 525-536.

69 Driesen (2004).

70 Sunstein (2018).

71 Driesen (2004).

72 Chauncey Starr, Social Benefit versus Technological Risk, in: Science 165, 1969, S. 1232-1238.

73 Man spricht hier auch von benefit transfers (Übertragung von Nutzen). Die in (kontingenten) Bewertungen gemessenen Werte für nicht monetäre Güter werden auf einen anderen Kontext übertragen.

74 Fraiberg/Trebilcock (1998). 
stellt sich die Frage, wie mit den Ergebnissen von Kosten-Nutzen-Analysen verfahren werden soll, wenn deren Ergebnisse gesellschaftlichen Konventionen widersprechen oder moralische Grenzen überschreiten. ${ }^{75}$ Dieses Problem moralischer Aufladung sachlicher Analyse zeigt sich insbesondere in Feldern, die nicht unabhängig von größeren politischen Problemlagen zu lösen sind. Die weltweite Bekämpfung von Krankheiten wie HIV oder Malaria etwa lässt sich nicht unabhängig von Fragen nach Gerechtigkeit und Verteilungskonflikten betrachten, so dass auch die dazugehörigen ökonomischen Berechnungen immer einen Kommentar oder sogar eine Handlungsempfehlung zum Problem der globalen Armut beinhalten.

Fazit

Dieses Kapitel zeigt, welche Bedeutung die Praktiken Bewerten und Gewichten im Feld der internationalen Gesundheits- und Umweltpolitik haben und welche Möglichkeiten und gleichzeitig Konflikte und Kontroversen diese zentrale Stellung der Kosten-Nutzen-Analyse eröffnet. Indem Entscheidungsprozesse in komplexen Problemlagen mit Kosten-NutzenAnalysen legitimiert werden, haben sie sich nach und nach als Schlüsselverfahren für Bewertungen und Gewichtungen in der evidenzbasierten Politik etabliert und damit alternative Bewertungsverfahren verdrängt. Sie dienen als Möglichkeit, diverse Interessen und Effekte durch Monetarisierung auf eine vergleichbare, argumentative Ebene zu stellen. Die Verwendung etablierter ökonomischer Verfahren ermöglicht idealerweise, den Ansprüchen an Bewertungs- und Gewichtungsprozesse in Bezug auf intersubjektive Nachvollziehbarkeit, Transparenz, Wiederholbarkeit und Glaubwürdigkeit gerecht zu werden. In den Beispielen des Kapitels zeigt sich, dass dies keinesfalls eine einfache Aufgabe ist. Die zunehmende Formalisierung des Verfahrens führt automatisch zum Ausschluss von bestimmten Faktoren der Anspruch an die Replizierbarkeit von Entscheidungen impliziert gleichzeitig einen Verlust der Anpassungsfähigkeit. Die Beispiele dieses Kapitels zeigen, wie anspruchsvoll die Forderung nach Evidenz als gemeinsame Basis für die Begründung von Entscheidungen sein kann. Die verschränkte Analyse von Bewertungs- und Gewichtungsprozessen hat zudem das Potential, die Bedeutung impliziter Faktoren sichtbar zu machen.

75 Sunstein nennt hier als Beispiel Tierwohl - auch wenn die Zahlungsbereitschaft niedrig wäre, ist es moralisch geboten, dass Tiere nicht misshandelt werden dürfen; Sunstein (2018). 
Die Komplexität der geschilderten Entscheidungen in der internationalen Umwelt- und Gesundheitspolitik erfordert eine stetige Aushandlung, wie Risikofaktoren und Folgeabschätzungen in die Entscheidungsfindung einbezogen werden sollen. Die Frage, wie Daten in diesen Auseinandersetzungen beurteilt werden und dadurch wertbasierte Urteile rechtfertigen und begründen, hat dabei auch eine politische Dimension: Von Bewertungen und Gewichtungen hängen weitreichende politische Entscheidungen, die Verteilung von Ressourcen und die Ausrichtung großangelegter Programme ab. Die Evidenzpraktiken des Bewertens und Gewichtens sind damit beides: Einerseits Machtinstrumente der Politik, andererseits Instrumente im Modus des Begründens und der Konfliktbewältigung.

\section{Literatur}

Adams, Vincanne, Metrics. What Counts in Global Health, Durham, NC 2016.

Arrow, Kenneth J., Eads, George C., Hahn, Robert W., Lave, Lester B., Noll, Roger G., Portney, Paul R., Russell, Milson, Schmalensee, Richard, Smith, V. Kerry u. Stavins, Robert N., Is There a Role for Benefit-Cost Analysis in Environmental, Health, and Safety Regulation?, in: Science, New Series Vol. 272, No. 5259, 1996, S. 221-22.

Babcock, Bruce A., Lichtenberg, Erik u. Zilberman, David, Impact of Damage Control and Quality of Output. Estimating Pest Control Effectiveness, in: American Journal of Agricultural Economics 74, 1992, S. 163-172.

Banerjee, Abhijit V. u. Duflo, Esther, Poor Economics. Plädoyer für ein neues Verständnis von Armut, München 2012.

Barnes, Amy u. Parkhurst, Justin, Can Global Health Policy be Depoliticized? A Critique of Global Calls for Evidence-Based Policy, in: Garrett Wallace Brown, Gavin Yamey u. Sarah P. Wamala (Hg.), The Handbook of Global Health Policy, Bd. 84, Chichester, West Sussex u. Malden, MA 2014, S. 157-173.

Brieger, William, Africa Health. The Challenge of Using and Misusing InsecticideTreated Bed Nets, in: Africa Health 39, 2017, S. 13-15.

Clinton, Chelsea u. Sridhar, Devi Lalita, Governing Global Health. Who Runs the World and Why?, New York, NY 2017.

Cropper, Maureen L., Evans, William N., Berardi, Stephen J., Ducla-Soares, Maria M. u. Portney, Paul R., The Determinants of Pesticide Regulation. A Statistical Analysis of EPA Decision Making, in: Journal of Political Economy 100, H. 1, 1992, S. 175-197.

Dobrow, Mark J., Goel, Vivek u. Upshur, Ross E. G., Evidence-Based Health Policy. Context and Utilisation, in: Social Science and Medicine 58, Heft 1, 2004, S. 207-217. 
Dolan, Carrie B., BenYishay, Ariel, Grépin, Karen A., Tanner, Jeffery C., Kimmel, April D., Wheeler, David C. u. McCord, Gordon C., The Impact of an Insecticide Treated Bednet Campaign on All-Cause Child Mortality. A Geospatial Impact Evaluation from the Democratic Republic of Congo, in: PLOS ONE 14, H. 2, 2019: https://doi.org/10.1371/journal.pone.0212890.

Driesen, David M., Distributing the Costs of Environmental, Health, and Safety Protection. The Feasability Principle, Cost-Benefit Analysis, and Regulatory Reform, in: Boston College Environmental Affairs Law Review 31, H. 1, 2004.

European Commission, EACEA u. Eurydice, Support Mechanisms for EvidenceBased Policy-Making in Education, Luxemburg 2017.

Florax, Raymond J. G. M., Travisi, Chiara M. u. Nijkamp, Peter, A Meta-Analysis of the Willingness to Pay for Reductions in Pesticide Risk Exposure, in: European Review of Agricultural Economics 32, H. 4, 2005, S. 441-467.

Fraiberg, Jeremy D. u. Trebilcock, Michael J., Risk Regulation. Technocratic and Democratic Tools for Regulatory Reform, in: McGill Law Journal 43, 1998, S. 835-888.

Fraser, Nicole, Partnerships for Malaria Control. Engaging the Formal and Informal Private Sectors; a Review Commissioned by the UNICEF/UNDP/World Bank/WHO, Genf 2006.

Goodman, Catherine A.; Coleman, Paul G. u. Mills, Anne J., Cost-Effectiveness of Malaria Control in Sub-Saharan Africa, in: The Lancet 354, H. 9176, 1999, S. 378-385.

Gray, George M. u. Hammitt, James K., Risk/Risk Trade-Offs in Pesticide Regulation. An Exploratory Analysis of the Public Health Effects of a Ban on Organophosphate and Carbamate Pesticides, in: Risk Analysis 20, H. 5, 2000, S. 665-680.

Guimarães, Raphael Mendonça, Asmus, Carmen Ildes Rodrigues Fróes u. Meyer, Armando, DDT Reintroduction for Malaria Control. The Cost-Benefit Debate for Public Health, in: Cadernos de saude publica 23, H. 12, 2007, S. 2835-2844.

Handford, Caroline E., Elliott, Christopher T. u. Campbell, K., A Review of the Global Pesticide Legislation and the Scale of Challenge in Reaching the Global Harmonization of Food Safety Standards, in: Integrated Environmental Assessment and Managment 11, 2015, H.4, S. 525-536.

Hayek, Friedrich August von, Die Anmaßung von Wissen. Neue Freiburger Studien, Tübingen 1996.

Hicks, John Richard, The Foundations of Welfare Economics, in: The Economic Journal 49, H. 196, 1939, S. 696-712.

Hill, Jenny, Lines, Jo u. Rowland, Mark, Insecticide-Treated Nets, in: D. H. Molyneux (Hg.), Control of Human Parasitic Diseases, New York 2007, S. 77128.

Kabasenche, William P. u. Skinner, Michael K., DDT, Epigenetic Harm, and Transgenerational Environmental Justice, in: Environmental Health. A Global Access Science Source 13, 2014, S. 62. 
Kaldor, Nicholas, Welfare Propositions of Economics and Interpersonal Comparisons of Utility, in: The Economic Journal 49, H. 195, 1939, S. 549-552.

Kawasaki, Kentaro u. Lichtenberg, Erik, Quality versus Quantity Effects of Pesticides. Joint Estimation of Quality Grade and Crop Yield, in: AAEA Annual Meeting, San Francisco 2015.

Kinchy, Abby J., Anti-Genetic Engineering Activism and Scientized Politics in the Case of "Contaminated" Mexican Maize, in: Agriculture and Human Values 27, H. 4, 2010, S. 505-517.

Klinke, Andreas u. Renn, Ortwin, A New Approach to Risk Evaluation and Management. Risk-Based, Precaution-Based, and Discourse-Based Strategies, in: Risk Analysis 22, H. 6, 2002, S. 1071-1094.

Kramer, Randall A.. Dickinson, Katherine. Anderson, Richard M.. Fowler, Vance G.. Miranda, Marie Lynn, Mutero, Clifford M. et al., Using Decision Analysis to Improve Malaria Control Policy Making, in: Health Policy 92, H. 2-3, 2009, S. $133-140$.

Krezanoski, Paul J., Delivering Insecticide-Treated Nets for Malaria Prevention. Innovative Strategies, in: Research and Reports in Tropical Medicine 7, 2016, S. 39-47.

Kouyaté, Bocar, Sie, Ali, Yé, Maurice, de Allegri, Manuela u. Müller, Olaf, The Great Failure of Malaria Control in Africa. A District Perspective from Burkina Faso, in: PLOS Medicine 4, H. 6, 2007, e127.

Lancaster, Kari, Seear, Kathryn Leigh, Treloar, Carla u. Ritter, Alison, The Productive Techniques and Constitutive Effects of "Evidence-Based Policy" and "Consumer Participation” Discourses in Health Policy Processes, in: Social Science and Medicine 176, 2017, S. 60-68.

Lynch, Michael P., In Praise of Reason, Cambridge, MA 2012.

Malaria Advocacy Working Group, Evidence for Advocacy. Key Statistics on the Fight Against Malaria. Roll Back Malaria Partnership, Genf 2015.

Marmot, Michael G., Evidence Based Policy or Policy Based Evidence?, in: British Medical Journal 328, April 2004, S. 906-907.

Merriam-Webster, Definition of Evaluate, 2019: https://www.merriam-webster.com /dictionary/evaluate [Stand: 22.6.2019].

Merriam-Webster, Definition of Weight, 2019: https://www.merriam-webster.com/ dictionary/weight [Stand: 22.6.2019].

Moore-Sheeley, Kirsten, Nothing but Nets. The History of Insecticide-Treated Nets in Africa, 1980s-Present. Dissertation Johns Hopkins University 2017.

Nabarro, David N. u. Tayler, Elizabeth M., The "Roll Back Malaria" Campaign, in: Science 280, H. 5372, 1998, S. 2067-2068.

Parks, Rachel, The Rise, Critique and Persistence of the DALY in Global Health, in: The Journal of Global Health, 2014: https://www.ghjournal.org/the-rise-criti que-and-persistence-of-the-daly-in-global-health/ [Stand: 21.8.2019]. 
Peterson, Robert K., Barber, Loren M. u. Schleier, Jerome J., Net Risk. A Risk Assessment of Long-Lasting Insecticide Bed Nets Used for Malaria Management, in: The American Journal of Tropical Medicine and Hygiene 84, H. 6, 2011, S. 951-956.

Pfeiffer, James u. Nichter, Mark, What Can Critical Medical Anthropology Contribute to Global Health?, in: Medical Anthropology Quarterly 22, H. 4, 2008, S. 410-415.

Porter, Theodore M., Trust in Numbers. The Pursuit of Objectivity in Science and Public Life, Princeton 1997.

Purdy, Mark, Robinson, Matthew, Wei, Kuangyi u. Rublin, David, The Economic Case for Combating Malaria, in: The American Journal of Tropical Medicine and Hygiene 89, H. 5, 2013, S. 819-823.

RBM Partnership to End Malaria, Annual Report 2017, S. 8 u. 11: https:/endmalari a.org/sites/default/files/RBM\%20Annual\%20Report_web.pdf [Stand: 22.6.2019].

RBM Partnership to End Malaria, Annual Report 2018, : https://endmalaria.org/site s/default/files/RBM\%20Annual\%20Report\%202018_EN.pdf [Stand: 22.6.2019].

RBM Partnership to End Malaria, RBM Partnership Strategic Plan 2018-2020, 2018, S. 13: https://endmalaria.org/sites/default/files/RBM-Strategic-Plan-digital-J W-220218.pdf [Stand: 22.6.2019].

Robinson, Lisa A., Hammitt, James K., Jamison, Dean T. u. Walker, Damian G., Conducting Benefit-Cost Analysis in Low- and Middle-Income Countries: Introduction to the Special Issue, in: Journal of Benefit-Cost Analysis, S1, 2019, S. 114.

Robinson, Lisa A., Hammitt, James K. u. O’Keeffe, Lucy, Valuing Mortality Risk Reductions in Global Benefit-Cost Analysis, in: Journal of Benefit-Cost Analysis 10, S1, 2019, S. 15-50.

Roll Back Malaria Partnership, Action and Investment to Defeat Malaria 20162030, 2015: https://endmalaria.org/sites/default/files/RBM_AIM_Report_0.pdf [Stand: 22.6.2019].

Roll Back Malaria Partnership, Multisectoral Action Framework for Malaria, 2015, https://endmalaria.org/sites/default/files/9_Multisectoral-Action-Framework-forMalaria.pdf [Stand: 22.6.2019].

Remme, Jan H., Binka, Fred u. Nabarro, David N., Toward a Framework and Indicators for Monitoring Roll Back Malaria, in: The American Journal of Tropical Medicine and Hygiene 64, 1-2 Suppl., 2001, S. 76-84.

Remme, Michelle, Martinez-Alvarez, Melisa u. Vassall, Anna, Cost-Effectiveness Thresholds in Global Health. Taking a Multisectoral Perspective, in: Value in Health. The Journal of the International Society for Pharmacoeconomics and Outcomes Research 20, H. 4, 2017, S. 699-704.

Roll Back Malaria Partnership, Evidence for Advocacy. Key Statitics on the Fight for Malaria, 2015: http://www.makingmalariahistory.org/wp-content/uploads/20 15/06/Malaria_Evidence-for-Advocacy_April_2015.pdf [Stand: 22.6.2019].

Saltelli, Andrea u. Giampietro, Mario, What Is Wrong with Evidence Based Policy, and How Can It Be Improved?, in: Futures 91, 2017, S. 62-71. 
Sexton, Steven E., Lei, Zhen u. Zilberman, David, The Economics of Pesticides and Pest Control, in: International Review of Environmental and Resource Economics 1, H. 3, 2007, S. 271-326.

Starr, Chauncey, Social Benefit versus Technological Risk, in: Science 165, 1969, S. $1232-1238$.

Statistisches Bundesamt, „Indikatoren für evidenzbasierte Politik“ 2019, Wiesbaden.

Stein, Felix u. Sridhar, Devi, The Financialisation of Global Health, in: Wellcome Open Research 3, 2018, S. 17.

Steketee, Richard W. u. Campbell, Carlos C., Impact of National Malaria Control Scale-up Programmes in Africa: Magnitude and Attribution of Effects, in: Malaria Journal 9, 2010, S. 299.

Sunstein, Cass R., The Cost-Benefit Revolution, Cambridge 2018.

Taebi, Behnam, Bridging the Gap between Social Acceptance and Ethical Acceptability, in: Risk Analysis 37, H. 10, 2017, S. 1817-1827.

Teklehaimanot, Awash u. Mejia, Paola, Malaria and Poverty, in: Annals of the New York Academy of Sciences 1136, 2008, S. 32-37.

Travisi, Chiara Maria, Nijkamp, Peter u. Vindigni, Gabriella, Pesticide Risk Valuation in Empirical Economics. A Comparative Approach, in: Ecological Economics 56, H. 4, 2006, S. 455-474.

Wagner, Markus, Law Talk v. Science Talk: The Languages of Law and Science in WTO Proceedings, in: Fordham International Law Journal 35, H. 1, 2016, S. 151-200.

Webb, James L. A., The Long Struggle Against Malaria in Tropical Africa, Cambridge 2014.

Yamey, Gavin, Global Campaign to Eradicate Malaria. Roll Back Malaria Has Achieved a High Profile But Little Real Action, in: British Medical Journal 322, 2001, S. 1191 f. 